\title{
Triagem neonatal: conhecimento dos alunos da graduação em enfermagem sobre o teste do pezinho
}

\section{Newborn screening: how undergraduate nursing students perceive the guthrie test}

\author{
Letícia Pinto Rodrigues ${ }^{1}$; Vanderlei José Haas ${ }^{2}$; Alessandra Bernadete Trovó \\ de Marqui ${ }^{3}$
}

\begin{abstract}
Resumo
Objetivo: Esse estudo teve por objetivo verificar o conhecimento dos alunos de enfermagem sobre o Teste do Pezinho, assim como sua forma de abordagem na graduação. Métodos: Trata-se de um estudo descritivo, transversal de abordagem quantitativa, com 168 acadêmicos de duas Instituições de Ensino Superior do município de Uberaba/Minas Gerais. Para a coleta foi utilizado um questionário e os dados foram analisados pelo Programa Statistical Package for the Social Sciences. Resultados: Quanto ao conhecimento sobre o Teste do Pezinho, os principais resultados foram: confusão do Teste do Pezinho com impressão plantar (12\%), comprometimento da etiologia das patologias diagnosticadas, equívoco quanto ao período e local para coleta do exame. Apenas cerca de $23 \%(\mathrm{~N}=38)$ responderam corretamente todas as patologias triadas pelo Teste do Pezinho no Estado de Minas Gerais. O tema Teste do Pezinho foi abordado principalmente em aulas expositivas $(67,4 \%)$ em disciplinas ofertadas nos semestres finais do curso de Graduação em Enfermagem, tais como: Assistência integral à saúde da criança e adolescente (41,5\%), Enfermagem em pediatria $(35,6 \%)$ e Enfermagem na saúde da criança $(33,3 \%)$. Conclusão: Os estudantes de enfermagem possuem conhecimento adequado acerca do TP, mas há lacunas em alguns temas pertinentes a esse exame, tais como período e local de coleta, etiologia, quais as doenças detectadas, entre outros.
\end{abstract}

Palavras chave: Triagem neonatal. Enfermagem. Educação. Estudantes. Conhecimento.

\begin{abstract}
Objective: This study aimed to determine what nursing students know about the Guthrie Test as well as how this topic is approached in undergraduate courses. Methods: Descriptive, cross-sectional, quantitative approach, including 168 students from two undergraduate institutions of the city of Uberaba/Minas Gerais. For data collection, a questionnaire was used, and the statistical analysis was made with the Statistical Package for Social Sciences software. Results: Regarding knowledge of the Guthrie Test, the main results were: confusion between Guthrie Test and footprints (12\%), impaired etiology establishment for diagnosed diseases, errors about the time and place of sample collection. Only about $23 \%$ of the students $(n=38)$ gave fully correct answers regarding the pathologies screened by the Guthrie Test. The theme Guthrie Test has been addressed mainly in lectures (67.4\%), as part of courses given in the final semester of the Nursing Graduation Course, such as: Comprehensive health care of children and adolescents (41.5\%), Nursing in pediatrics $(35.6 \%)$, and Nursing in child health $(33.3 \%)$. Conclusion: The nursing students have adequate
\end{abstract}

\footnotetext{
1 Acadêmica de Enfermagem da UFTM, Uberaba/MG. Bolsista de Iniciação Científica da Fundação de Amparo à Pesquisa de Minas Gerais/FAPEMIG. E-mail: leticiapinto.rodrigues@hotmail.com

2 Doutor em Física Aplicada à Medicina e Biologia pela Universidade de São Paulo, Pós-doutorado pela Faculdade de Medicina USP. Professor visitante da UFTM, Uberaba/MG. E-mail: haasbiostat2010@enfermagem.uftm.edu.br

3 Bióloga. Doutora em genética pela Universidade Estadual Paulista Júlio de Mesquita Filho - UNESP. Professora associada da disciplina de Genética da UFTM, Uberaba/MG. E-mail: alessandratrovo@hotmail.com
} 
knowledge regarding the Guthrie Test, but there are gaps in some topics related to this test, such as time and place of collection, etiology, which diseases are detected among others.

Keyword: Neonatal screening. Nursing. Education. Students. Knowledge.

\section{Introdução}

A Triagem Neonatal (TN) foi implantada no Brasil em 1976 pela Associação de Pais e Amigos dos Excepcionais (APAE/SP) para diagnóstico de fenilcetonúria (PKU). A partir de 1980 foi incluído a triagem para hipotireoidismo congênito (HC) e na década de 90, a triagem para essas duas patologias ficou conhecida como teste do pezinho (TP). Nesse exame, algumas gotas de sangue são obtidas do calcanhar do recém-nascido $(\mathrm{RN})$, por ser uma região altamente vascularizada e praticamente indolor para o bebê e, depositadas em papel filtro. Em 2001, o governo federal implantou por meio da portaria $n^{\circ} 822$ de 06 de junho, o Programa Nacional de Triagem Neonatal (PNTN), que estabelece que toda criança nascida em território nacional deve ser submetida ao TP, que é gratuito e obrigatório (BRASIL, 2001). Através da lei $\mathrm{n}^{\mathrm{o}} 11.605$, de 5 de dezembro de 2007, foi instituído a data de 6 de junho como o dia nacional do TP (BRASIL, 2007).

O TP faz parte de um dos testes inclusos na TN que abrange também os testes da orelhinha (BRASIL, 2004a), dos olhinhos (BRASIL, 2011), da linguinha (BRASIL, 2014a) e do coraçãozinho (BRASIL, 2014b), que são importantes para diagnosticar distúrbios que possam prejudicar o desenvolvimento do RN. Esse exame é realizado no período neonatal e sua finalidade é a detecção precoce de doenças denominadas erros inatos do metabolismo. No Estado de Minas Gerais, as seguintes patologias são diagnosticadas pelo PNTN: PKU, HC, anemia falciforme e outras hemoglobinopatias, fibrose cística, hiperplasia adrenal congênita e deficiência de biotinidase (BRASIL, 2012). Essas doenças não possuem cura, mas exibem um bom prognóstico se tratadas desde o período neonatal. Vale destacar que a
TN é o maior programa de saúde pública ligada à genética no Brasil e constitui uma estratégia de prevenção, principalmente, da deficiência intelectual (LEÃO; AGUIAR, 2008).

A realização do TP é um procedimento de enfermagem, na qual o enfermeiro ou técnico de enfermagem são os profissionais capacitados para realização da coleta. O período para realizar o exame deve ser do $3^{\circ}$ ao $7^{\circ}$ dia de vida do $\mathrm{RN}$, preferencialmente no $5^{\circ}$ dia, pois o mesmo já esteve em contato com o leite materno, que é fonte de alimentação protéica. É importante que o profissional de saúde informe aos pais que caso o teste dê positivo deve-se fazer o exame diagnóstico, pois a TN não é confirmatória, assim pode haver falsos-positivos e falsos-negativos (BRASIL, 2004a).

Um dos estudos publicados na literatura mostrou que os profissionais da equipe de enfermagem da unidade de terapia intensiva neonatal de um hospital público do interior paulista não associaram o $\mathrm{TP}$ com a $\mathrm{TN}$, não sabiam os nomes das patologias triadas, o período preconizado para a coleta e outras informações pertinentes ao teste (BENINCASA et al., 2009). Deste modo, os estudos têm investigado o papel da enfermagem no TP, com enfoque no conhecimento desses profissionais e no procedimento de coleta deste exame (ACOSTA; STREFLING; GOMES, 2013; BENINCASA et al., 2009; STREFLING et al., 2014; PIMENTE et al., 2010). Essa fragilidade quanto à atuação da equipe de enfermagem em educação em saúde pode ter refletido diretamente no conhecimento superficial dos pais sobre esse exame (ABREU; BRAGUINI, 2011; SALLES; SANTOS, 2009; SANTOS et al., 2011).

Não há pesquisas sobre o conhecimento dos estudantes de enfermagem, futuros profissionais 
de saúde sobre o tema. Assim, é fundamental conhecer o que sabem os universitários de enfermagem acerca do TP para rever conteúdos curriculares e estratégias pedagógicas para o ensino do tema. Portanto, esse estudo tem por objetivo verificar o conhecimento dos alunos de enfermagem sobre o TP, assim como sua forma de abordagem na graduação.

\section{Métodos}

Trata-se de um estudo descritivo, transversal de abordagem quantitativa. Foram investigados 168 acadêmicos de enfermagem de duas Instituições de Ensino Superior (IES) do município de Uberaba/MG. A IES federal foi denominada 1 e a particular, 2. A IES 1 oferece inúmeros cursos de graduação e é referência em ensino público no Triângulo Mineiro, com destaque para a graduação em enfermagem em nível nacional. A instituição particular é a maior do município e possui um hospital universitário recém-construído para os cursos da área da saúde.

Foram incluídos na pesquisa alunos regularmente matriculados nos cursos de graduação em enfermagem de todos os períodos das IES propostas, que estavam presentes em sala de aula no dia de aplicação do questionário e que aceitaram participar voluntariamente da pesquisa.

A coleta de dados foi realizada durante o período de setembro a novembro de 2014. Com a autorização prévia do coordenador do curso e do professor presente em sala de aula, os objetivos da pesquisa foram informados aos alunos presentes e, aqueles que concordaram em participar assinaram o Termo de Consentimento Livre Esclarecido e receberam o questionário para responder. O questionário foi dividido em duas partes: I) dados dos estudantes de graduação e II) conhecimento dos alunos sobre o TP. Em relação ao item I do questionário, as variáveis analisadas foram instituição de origem, turno, idade, sexo, estado civil, segundo grau público ou privado, formação técnica em enfermagem e atuação profissional na saúde. As questões específicas do conhecimento sobre o tema da pesquisa foram fechadas, de múltipla escolha com a indicação de informar apenas uma alternativa correta. Esse instrumento foi elaborado pelas próprias autoras e preenchido pelos participantes.

Os dados foram tabulados e analisados por meio de estatística descritiva em frequências absolutas e percentuais com os Programas Microsoft Office Excel e Statistical Packaje for the Social Sciences (SPSS) versão 21.0.

O presente estudo foi aprovado pelo Comitê de Ética em Pesquisa com Seres Humanos da Universidade Federal do Triângulo Mineiro/ UFTM (número do parecer 859.344) e atendeu os preceitos éticos estabelecidos pela Resolução 466/2012 do Conselho Nacional de Saúde.

\section{Resultados}

Dos 168 acadêmicos de enfermagem, 92 pertenciam à IES 1 (turno integral) e 76 à IES 2 (turnos integral $n=53$ e noturno $n=23$ ).

Quanto à caracterização dos estudantes de enfermagem investigados, a maioria é do sexo feminino, solteiras, egressas do ensino médio de escola pública e quase $21 \%$ tinham o curso técnico em enfermagem (Tabela 1). 
Tabela 1 - Perfil dos estudantes da graduação em enfermagem investigados, Uberaba-MG, 2014

\begin{tabular}{cccc}
\hline Variáveis & & $\mathbf{n}$ & $\mathbf{\%}$ \\
\hline Sexo & Feminino & 141 & 84,9 \\
Estado civil & Masculino & 25 & 15,1 \\
& Casado (a) & 17 & 10,2 \\
& Solteiro (a) & 147 & 88,0 \\
& Divorciado (a) & 1 & 0,6 \\
Ensino médio & Mora com companheiro(a) & 2 & 1,2 \\
& Escola pública & 121 & 73,0 \\
Trabalha na área da saúde & Escola particular & 45 & 27,0 \\
UBS/ILPI/LAC/CD/Outro & Sim & 34 & 20,7 \\
\hline
\end{tabular}

UBS (Unidade Básica de Saúde), ILPI (Instituição de Longa Permanência para Idosos), LAC (Laboratório de Análises Clínicas) e CD (Cuidador Domiciliar).

Fonte: Autor

Com relação aos conhecimentos dos graduandos sobre o TP, os principais resultados quanto ao conhecimento dos estudantes sobre o TP foram: comprometimento da etiologia das doenças detectadas pelo TP; confusão do teste com impressão plantar realizada antes da alta hospitalar; indicação incorreta para coleta do exame nas primeiras 48 horas de vida, o que interfere no resultado para triagem de PKU. Ficou evidente que a coleta e a orientação sobre o TP é de competência da enfermagem; que esse exame é obrigatório; sem risco para o bebê e o sistema público de saúde é referência para sua realização. Quanto às orientações, foi destacada a sua realização nos diversos momentos, o que é adequado. Os alunos indicaram que um dos principais motivos para não realização do TP no bebê por parte da mãe é a falta de informação. Nesse sentido, estes graduandos, futuros profissionais de saúde poderão orientar as mães adequadamente sobre o TP, conscientizandoas da importância da realização do mesmo.

Aproximadamente 80 e $82 \%$ dos acadêmicos ouviram falar sobre $\mathrm{TP}$ e $\mathrm{TN}$, respectivamente na faculdade/universidade $(63 \%)$ e por meios de comunicação, como televisão, rádio e internet referido por $49 \%$ dos participantes. 
Tabela 2 - Conhecimento dos estudantes de graduação em enfermagem sobre o Teste do Pezinho, Uberaba-MG, 2014.

\begin{tabular}{|c|c|c|c|}
\hline \multicolumn{2}{|l|}{ Variáveis } & $\mathbf{n}$ & $\%$ \\
\hline \multirow{3}{*}{ Qual a finalidade do teste? } & Saber se a criança tem alguma doença & 90 & 57,3 \\
\hline & Saber se a criança terá alguma doença genética & 61 & 38,9 \\
\hline & Não sei & 6 & 3,8 \\
\hline \multirow{3}{*}{ Qual a importância do TP? } & Detecção precoce de algumas doenças tratáveis & 141 & 89,2 \\
\hline & $\begin{array}{c}\text { Detecçã̃o precoce de algumas doenças não } \\
\text { tratáveis }\end{array}$ & 11 & 7,0 \\
\hline & Não sei & 6 & 3,8 \\
\hline \multirow{3}{*}{$\begin{array}{l}\text { Você sabe como o teste é } \\
\text { realizado? }\end{array}$} & $\begin{array}{l}\text { Coleta de sangue apenas do pé do recém- } \\
\text { nascido }\end{array}$ & 157 & 95,2 \\
\hline & Carimbo do pé da criança & 20 & 12,1 \\
\hline & Coleta de sangue do braço por punção venosa & 6 & 3,6 \\
\hline \multirow{4}{*}{$\begin{array}{c}\text { Realização do TP é atribuição } \\
\text { do(a) }\end{array}$} & Equipe de enfermagem & 155 & 95,1 \\
\hline & Não sei & 5 & 3,1 \\
\hline & Médico & 2 & 1,2 \\
\hline & Agente de saúde & 1 & 0,6 \\
\hline \multirow{4}{*}{ Orientação sobre TP pelo(a) } & Equipe de enfermagem & 111 & 77,1 \\
\hline & Médico Pediatra & 23 & 16,0 \\
\hline & Médico obstetra & 5 & 3,5 \\
\hline & Não sei & 5 & 3,5 \\
\hline \multirow{3}{*}{$\begin{array}{l}\text { O teste traz algum risco para } \\
\text { o bebê? }\end{array}$} & Não & 158 & 98,1 \\
\hline & Sim & 2 & 1,2 \\
\hline & Não sei & 1 & 0,6 \\
\hline \multirow{5}{*}{ O teste deve ser realizado? } & Primeiras 48 horas de vida & 37 & 22,8 \\
\hline & Do $3^{\circ}$ ao $7^{\circ}$ dia de vida & 105 & 64,8 \\
\hline & Do $8^{\circ}$ ao $11^{\circ}$ dia de vida & 7 & 4,3 \\
\hline & Do $11^{\circ}$ ao $30^{\circ}$ dia de vida & 1 & 0,6 \\
\hline & Não sei & 12 & 7,4 \\
\hline \multirow{3}{*}{ O TP é um exame } & Obrigatório & 126 & 77,3 \\
\hline & Facultativo & 30 & 18,4 \\
\hline & Não sei & 7 & 4,3 \\
\hline \multirow{2}{*}{$\begin{array}{c}\text { O TP é um exame realizado } \\
\text { pelo }^{\mathrm{a}}\end{array}$} & Sistema público custeado pelo SUS & 160 & 97,0 \\
\hline & Sistema particular & 74 & 44,8 \\
\hline \multirow{3}{*}{ Momento para orientar o TP?a } & Pré-natal & 114 & 69,1 \\
\hline & Alta hospitalar/maternidade & 110 & 66,7 \\
\hline & UBS no momento da realização do teste & 81 & 49,1 \\
\hline \multirow{4}{*}{$\begin{array}{l}\text { Motivos das mães não } \\
\text { realizarem o TP?a }\end{array}$} & Desinformação acerca do teste & 136 & 82,4 \\
\hline & Acham o procedimento doloroso & 99 & 60,0 \\
\hline & As mães não acham o teste importante & 74 & 44,8 \\
\hline & Religião & 10 & 6,1 \\
\hline
\end{tabular}

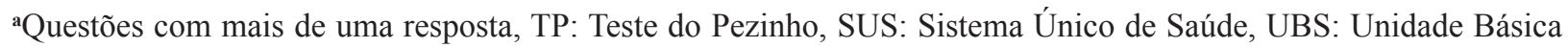
de Saúde

Fonte: Autor 
Em relação às doenças diagnosticadas, o resultado foi parcialmente satisfatório. Do total de estudantes, 58 responderam não saber quais as patologias diagnosticadas pelo TP e 39 não assinalaram nenhuma resposta. Doze estudantes citaram corretamente de uma a três patologias diagnosticadas por esse exame. Oito e treze acadêmicos citaram corretamente quatro e cinco patologias, respectivamente. Aproximadamente 23\% responderam corretamente todas as seis patologias triadas pelo TP no estado de MG. A maioria desses estudantes era provenientes dos períodos finais dos cursos de graduação em enfermagem. Entretanto, outras doenças tais como: hemofilia $(18,4 \%)$, Síndrome de Down (12,3\%), hepatite $(9,8 \%)$ e AIDS/Síndrome da Imunodeficiência Adquirida $(9,2 \%)$ foram citadas como sendo diagnosticadas pelo TP, mas essa informação não está correta.

No ensino superior, o tema TP foi abordado principalmente em aulas expositivas $(67,4 \%)$ de disciplinas ofertadas nos semestres finais do curso de graduação em enfermagem. As disciplinas referidas pelos acadêmicos que mais abordaram esse tema foram: Assistência integral à saúde da criança e adolescente (41,5\%), Enfermagem em pediatria $(35,6 \%)$ e Enfermagem na saúde da criança $(33,3 \%)$. Foi sugerido aumentar a carga horária das disciplinas que abordavam esse assunto por $30 \%$ dos entrevistados e aproximadamente $25 \%$ referiram a criação de uma disciplina optativa ou liga acadêmica sobre o TP.

\section{Discussão}

Os poucos estudos sobre TP foram realizados com profissionais de enfermagem (ACOSTA; STREFLING; GOMES, 2013; BENINCASA et al., 2009; PIMENTE et al., 2010; STREFLING et al., 2014), sendo apenas três sobre o conhecimento desses profissionais (ACOSTA; STREFLING; GOMES, 2013; BENINCASA et al., 2009; STREFLING et al., 2014). A abordagem do conhecimento dos acadêmicos é imprescindível pois, de posse de conhecimentos sólidos ele atuará de forma segura e adequada na sua prática profissional e, consequentemente contribuirá para a promoção da saúde e bem estar daquela família. A escassez de estudos pode ter limitado de alguma forma a discussão dos dados encontrados nessa pesquisa. Não há estudos publicados com estudantes de graduação, o que evidencia o ineditismo de nosso trabalho.

Entre os estudantes analisados, houve predomínio do sexo feminino. Esse achado é esperado tendo em vista que a enfermagem tem como principal característica o ato de cuidar e esse é frequentemente realizado pela mulher. Estudos recentes, com acadêmicos de enfermagem, mostraram frequências de aproximadamente 90\% de mulheres (NARDELLI et al., 2013; FREIRE; VASCONCELOS, 2013; PATRÍCIO et al., 2015). Nessa pesquisa, a frequência do estado civil solteiro foi abaixo daquela descrita na literatura, que referiu um valor ainda maior $(97,6 \%)$ entre os alunos ingressantes em cursos de graduação da área da saúde da mesma instituição de ensino (NARDELLI et al., 2013). A explicação para uma minoria trabalhar na área de saúde é a realização da graduação em turno integral.

Os estudantes pesquisados souberam diferenciar TN e TP. Esse achado contradiz aquele da literatura, pois dezenove profissionais da equipe de enfermagem de um total de 21 exibiram entendimento equivocado na definição desses termos (BENINCASA et al., 2009).

Apesar de saberem a finalidade do teste, a etiologia da doença ficou comprometida. A maioria das doenças detectadas pelo TP tem etiologia genética e, portanto há um risco de recorrência nas famílias. Um estudo com 171 profissionais da equipe de enfermagem que atuavam em unidades materno-infantis revelou despreparo e necessidade de capacitação em relação aos distúrbios genéticos (CUNHA et al., 2010). No entanto, foi destacado que o teste é importante para detecção precoce de doenças passíveis de tratamento. Esse resultado 
contradiz aquele relatado por Benincasa et al. (2009) pois nenhum dos 21 entrevistados citou a real importância da TN, que por sua vez é a prevenção de complicações que as patologias triadas ocasionam à vida da criança se, contudo, não diagnosticadas e tratadas precocemente.

Cerca de $12 \%$ dos estudantes confundiram o TP com a impressão plantar do bebê, colhida ao nascimento. Esse achado corrobora aquele presente na literatura (SANTOS et al., 2011). Um outro achado inesperado foi que somente cerca de $4 \%$ citaram a punção venosa como forma de obtenção do material biológico para a realização do exame. Isso mostra a necessidade de maior esclarecimento quanto à possibilidade de outros locais para coleta de sangue periférico. No entanto, o local indicado é a região lateral do calcanhar do pé por ser indolor ao bebê e muito vascularizada (BRASIL, 2004a).

Os estudantes sabem que a realização e a orientação do TP são atribuições da enfermagem. Esse último resultado reforça o papel dessa profissão na educação em saúde. $O$ enfermeiro como educador e prestador de uma assistência humanizada deve orientar e esclarecer as dúvidas das mães sobre o TP. Devem também enfatizar que esse exame é importante para a saúde do bebê por permitir diagnosticar doenças que poderão levar a deficiência intelectual. Alguns estudos relataram que a orientação sobre esse exame também foi prestada por médicos (SALLES; SANTOS, 2009; SANTOS et al., 2011; DELVIVO et al., 2012).

Em relação à idade para coleta do TP, o Ministério de Saúde preconiza como ideal do $3^{\circ}$ ao $7^{\circ}$ dia de vida do $\mathrm{RN}$, é aceitável do $8^{\circ}$ ao $30^{\circ}$ dia e acima de 30 dias considerado inapropriado (BRASIL, 2004a). Condições específicas tais como idade inadequada para coleta, prematuridade, dieta, transfusões e nutrição parenteral total podem influenciar os resultados do exame (LEÃO; AGUIAR, 2008). Apesar de 65\% indicarem o período correto, cerca de $25 \%$ responderam que a coleta deve ser nas primeiras 48 horas. Esse dado é preocupante, pois para triagem de PKU é necessário que o neonato já tenha recebido leite suficiente para que haja acúmulo de fenilalanina no sangue, caso seja afetado (BRASIL, 2004a). Conhecimento equivocado quanto ao período indicado para realizar a coleta também foi relatado em dois estudos com enfermeiras (ACOSTA; STREFLING; GOMES, 2013; BENINCASA et al., 2009), sendo que em um deles nenhum dos 21 entrevistados respondeu de forma completa o período preconizado para coleta (BENINCASA et al., 2009). Nesse sentido é necessário um maior aprofundamento sobre o período ideal para coleta do TP. Alguns autores sugerem a adoção do $3^{\circ}$ dia de vida do RN como sendo o "Dia do TP", pois essa estratégia contribuíra para uma divulgação mais abrangente e ampliaria a atenção desse programa de fundamental importância para a saúde pública (MAGALHÃES et al., 2009).

Os estudantes sabem da obrigatoriedade do exame e citaram o sistema público de saúde como referência para sua realização. Na graduação em enfermagem, as políticas públicas com enfoque no SUS (Sistema Único de Saúde) são amplamente discutidas, fato que explica o resultado prévio. A realização do TP no sistema público garante a família o tratamento adequado em um Serviço de Referência em Triagem Neonatal (SRTN), existente em cada estado brasileiro, que é composto por uma equipe multidisciplinar para o atendimento e acompanhamento da criança afetada, em caso de resultado positivo no teste confirmatório.

Quanto às orientações sobre o TP, os resultados ressaltaram a sua realização nos diversos momentos, o que é adequado. Segundo a literatura, a orientação deve se iniciar no pré-natal, pois, nesse período a mulher está mais atenta e tem condições de assimilar melhor essas informações (AL-ALAM et al., 2012). Durante a gravidez as mães possuem tempo para elaborar as informações recebidas, questioná-las, para posteriormente pô-las em prática (ACOSTA; STREFLING; GOMES, 2013). No pós-parto, a eficácia pode ser comprometida em virtude das mães estarem cansadas e em processo de adaptação com 
as necessidades de seu filho e de seu próprio corpo (DELVIVO et al., 2012). O momento da coleta, por sua vez, é a ocasião oportuna para a enfermeira reforçar as orientações sobre o TP (STREFLING et al., 2014). Assim, o ideal é que as informações sobre TP façam parte do ciclo gravídico puerperal. Apesar de aproximadamente $70 \%$ dos estudantes citarem que as informações devem ser repassadas as mães antes mesmo do nascimento do bebê, esse dado contradiz aquele relatado na literatura. Um desses estudos com 42 gestantes mostrou que apenas três $(7 \%)$ receberam orientações sobre o TP do médico durante o pré-natal $(\mathrm{n}=2)$ e uma em um cursinho para gestantes (OLIVEIRA et al., 2008). Em outro, realizado com treze enfermeiras atuantes nas unidades da Estratégia de Saúde da Família no Rio Grande do Sul, apenas uma enfermeira abordou o tema no pré-natal (ACOSTA; STREFLING; GOMES, 2013).

Os alunos indicaram que um dos principais motivos para não realização do TP no bebê por parte da mãe é a falta de informação, seguido pelo fato do exame ser doloroso para o bebê. Vale destacar que, para o manejo da dor podem ser utilizados métodos não farmacológicos no $\mathrm{RN}$, o que garantiria um cuidado qualificado e humanizado. Tais métodos incluem: sucção nutritiva (glicose/sacarose) e não nutritiva, amamentação, contato pele a pele, enrolamento, mudança de decúbito, reduzir a manipulação e os estímulos ambientais (ruídos e luminosidade), agrupar os cuidados e promover períodos de sono (CORDEIRO; COSTA, 2014; MOTTA; CUNHA, 2015; TASSINARY; HAHN, 2013). Estudo prévio confirmou que $55 \%$ das gestantes relataram que o motivo para não realização do TP foi o caráter doloroso do procedimento (OLIVEIRA et al., 2008). Esse resultado enfatiza a necessidade de maior divulgação do TP visando mostrar aos pais os benefícios que esse teste traz para o desenvolvimento do RN. Nesse sentido, tais graduandos, futuros profissionais de saúde, poderão conscientizar e sensibilizar os pais da importância da realização do mesmo.
Estudo prévio com discentes de um curso técnico de enfermagem de uma escola particular de $\mathrm{MG}$ mostrou que $50 \%$ deles têm interesse nas áreas de pediatria $(30,76 \%)$ e maternidade $(19,23 \%)$ como possíveis áreas de atuação (COSTA; BORGES; DONOSO, 2013). Dada a íntima relação desse profissional com o neonato e a mãe, respectivamente, é necessário um empoderamento de conhecimento técnico-científico na área de $\mathrm{TN}$, o que justifica analisar esse tema na graduação em Enfermagem. Vale ainda destacar que, esse tema merece grande destaque na formação do profissional de enfermagem pela sua importância e impacto direto na saúde da criança. Desse modo, esse profissional tem papel inquestionável na efetividade da $\mathrm{TN}$, pois atuará na prevenção de sequelas irreversíveis como a deficiência intelectual, contribuindo para a promoção da saúde.

Quanto às doenças investigadas pelo TP, algumas daquelas citadas pelos estudantes não são detectadas por esse exame. Esse resultado também foi relatado previamente sendo que cinco dos 21 profissionais da equipe de enfermagem incluíram patologias não passíveis de serem triadas pelo teste como hepatite, HIV e Síndrome de Down (BENINCASA et al., 2009). A hemofilia e a Síndrome de Down possuem etiologia genética e essa última foi referida por $99 \%$ dos profissionais de enfermagem investigados como o principal distúrbio genético conhecido por eles (CUNHA et al., 2010).

O tema TP foi abordado principalmente em aulas expositivas de disciplinas ministradas em períodos finais. Essa temática deveria ser desenvolvida com diferentes estratégias de ensino ao longo de toda a formação acadêmica, não se restringindo apenas ao término do curso. A abordagem do tema na graduação foi realizada em disciplinas com enfoque na saúde materno-infantil, com predomínio do estilo tradicional de ensino caracterizado por aulas expositivas. Vale destacar que, o objetivo principal do estudo foi verificar o conhecimento dos acadêmicos de enfermagem sobre o TP e a forma de abordagem na graduação foi resultado secundário. Portanto, uma pesquisa adicional 
poderia ser conduzida com a finalidade de avaliar o ensino dessa temática na graduação.

As limitações desse estudo foram o desenho transversal que representa um recorte da realidade em um determinado período de tempo e os viéses de informação por erros de memórias dos estudantes investigados. Em virtude da escassez de estudos com esse enfoque na literatura, é necessária a realização de outras pesquisas que possam auxiliar no conhecimento e ensino de TN e TP nos cursos de graduação de nosso país.

\section{Conclusão}

Os estudantes de enfermagem possuem conhecimento adequado acerca do TP, mas há lacunas em alguns temas pertinentes a esse exame, tais como período e local de coleta, etiologia, quais as doenças detectadas, entre outros. Nesse sentido, fazse necessário a implantação de ações com o intuito de esclarecer esses impasses tendo em vista que serão futuros profissionais de saúde que poderão atuar na área de cuidado à saúde da mãe e recém-nascido.

\section{Referências}

ABREU, I. S.; BRAGUINI, W. L. Triagem neonatal: o conhecimento materno em uma maternidade no interior do Paraná, Brasil. Revista Gaúcha de Enfermagem, Porto Alegre, v. 32, n. 3, p. 596-601, 2011.

ACOSTA, D. F.; STREFLING, I. S. S.; GOMES, V. L. Triagem neonatal: (re)pensando a prática de enfermagem. Revista de Enfermagem UFPE, Recife, v. 7, n. 2, p. 572-578, 2013.

AL-ALAM, A. C.; SOARES, M. C.; MEINCKE, S. M. K.; DILÉLIO, A. S.; ESCOBAL, A. P. L. Entendimento das Mães acerca da triagem neonatal: um estudo qualitativo. Journal of Nursing Health, Pelotas, v. 1, n. 2, p. 75-81, 2012.

BENINCASA, T. O.; OLIVEIRA, C. B.; ZANONI, I. H.; LIMA, S. A. O.; MARTINS, D. C. Triagem neonatal: a percepção teórica da equipe de enfermagem da unidade de terapia intensiva neonatal. Revista do Instituto de Ciências da Saúde, São Paulo, v. 27, n. 2 , p. 109-114, 2009.
BRASIL. Lei $n^{o} 11.605$, de 5 de dezembro de 2007. Institui o Dia Nacional do Teste do Pezinho a ser comemorado no dia 6 de junho de cada ano. Disponível em: <http:/www2.camara.leg.br/ legin/fed/lei/2007/lei-11605-5-dezembro-2007565687-norma-pl.html>. Acesso em: 14 nov. 2016.

BRASIL. Lei $n^{\circ}$ 13.002, de 20 de junho de 2014. Obriga a realização do Protocolo de Avaliação do Frênulo da Língua em Bebês. 2014a. Disponível em: <http://www.planalto.gov.br/ccivil_03/ Ato2011-2014/2014/Lei/L13002.htm>. Acesso em: 14 nov. 2016.

BRASIL. Ministério da Saúde. Manual de normas técnicas e rotinas operacionais do programa nacional de triagem neonatal. Brasília: Ministério da Saúde, 2004a.

BRASIL. Portaria $n^{\circ} 650$, de 5 de outubro de 2011. Institui no âmbito do SUS a Rede Cegonha. Disponível em: < http://www.redeblh.fiocruz.br/ media/port_650.pdf $>$. Acesso em: 14 nov. 2016.

BRASIL. Portaria no. 2.073, de 28 de setembro de 2004. Institui a Política Nacional de Atenção à Saúde Auditiva. 2004b. Disponível em: <http:// www.saude.mg.gov.br/images/documentos/ PORTARIA_2073.pdf $>$. Acesso em: 14 nov. 2016.

BRASIL. Portaria no. 2.829, de 14 de dezembro de 2012. Inclui a fase IV no Programa Nacional de Triagem Neonatal (PNTN), instituído pela Portaria no. 822/GM/MS, de 6 de junho de 2001. Disponível em: < http://www.cib.rj.gov. br/arquivos-para-baixar/portarias-cib/1122portaria-n-2-829-de-14-de-dezembro-de-2012/ file.html>. Acesso em: 14 nov. 2016

BRASIL. Portaria no. 20, de 10 de junho de 2014. Torna pública a decisão de incorporar a oximetria de pulso - teste do coraçãozinho, a ser realizado de forma universal, fazendo parte da triagem Neonatal no Sistema Único de Saúde - SUS. 2014b. Disponível em: <http:// www.poderesaude.com.br/novosite/images/ publicacoes_11.06.2014-III.pdf>. Acesso em: 14 nov. 2016. 
BRASIL. Portaria no. 822, de 06 de junho de 2001. Institui, no âmbito do Sistema Único de Saúde, o Programa Nacional de Triagem Neonatal - PNTN. Disponível em: <http://www.saude.mg.gov.br/images/ documentos/PORTARIA_822.pdf $>$. Acesso em: 14 nov. 2016.

CORDEIRO, R. A.; COSTA, R. Métodos não farmacológicos para alívio do desconforto e da dor no recém-nascido: uma construção coletiva da enfermagem. Texto \& Contexto Enfermagem, Florianópolis, v. 23, n. 1, p. 185-192, 2014.

COSTA, F. C.; BORGES, E. L.; DONOSO, M. T. V. Perfil dos alunos de curso técnico de enfermagem de uma escola particular em Minas Gerais. Revista de Enfermagem do Centro Oeste Mineiro, Divinópolis, v. 3, n. 1, p. 554-568, 2013.

CUNHA, V. M. P.; SILVA-GRECCO, R. L.; SILVA, S. R.; BALARIN, M. A. S. Conhecimento da equipe de enfermagem de unidades materno-infantis frente aos distúrbios genéticos. Revista da Rede de Enfermagem do Nordeste, Fortaleza, v. 11, n. esp., p. 215-222, 2010.

DELVIVO, E. M.; NAZARETH, J. B.; SALVADOR, M.; SALIMENA, A. M. O.; DIAS, I. M. A. V. Teste do pezinho: desvelando o conhecimento das mães sobre o exame. HURevista, Juiz de Fora, v. 38, n. 1, p. 45-50, 2012.

FREIRE, A. C. S.; VASCONCELOS, H. C. A. Doação de sangue: conhecimento, prática e atitude de acadêmicos de enfermagem de uma instituição do interior do Ceará. Revista Mineira de Enfermagem, Belo Horizonte, v. 17, n. 2, p. 296-303, 2013.

LEÃO, L. L.; AGUIAR, M. J. B. Triagem neonatal: o que os pediatras deveriam saber. Jornal de Pediatria, Rio de Janeiro, v. 84, n. 4, p. S80-S90, 2008.

MAGALHÃES, P. K. R.; TURCATO, M. F.; ANGULO, I. L.; MACIEL, L. M. Z. Programa de triagem neonatal do Hospital das Clínicas da Faculdade de Medicina de Ribeirão Preto, Universidade de São Paulo, Brasil. Cadernos de Saúde Pública, Rio de Janeiro, v. 25, n. 2, p. 445-454, 2009.

MOTTA, G. C. P.; CUNHA, M. L. C. da. Prevenção e manejo não farmacológico da dor no recém-nascido. Revista Brasileira de Enfermagem, Brasília, v. 68, n. 1, p. 131-135, 2015.
NARDELLI, G. G.; GAUDENCI, E. M.; GARCIA, B. B. G.; CARLETO, C. T.; GONTIJO, L. M.; PEDROSA, L. A. K. Perfil dos alunos ingressantes dos cursos da área da saúde de uma universidade federal. Revista de Enfermagem e Atenção à Saúde, Uberaba, v. 2, n. 1, p. 3-12, 2013.

OLIVEIRA, J. G.; SANDRINI, D.; COSTA, D. C.; SERRADILHA, A. F. Z.; PARRO, M. C. Triagem neonatal ou teste do pezinho: conhecimento, orientações e importância para a saúde do recémnascido. CuidArte Enfermagem, Bucaramanga, v. 2, n. 1, p. 71-76, 2008.

PATRÍCIO, A. C. F. A.; ALVES, K. L.; SANTOS, J. S.; ARARUNA, P.C.;DUARTE, M.C. S.; RODRIGUES, M. M. D. Exame físico cardiorrespiratório: conhecimento de estudantes de enfermagem. Revista de Pesquisa: Cuidado é Fundamental Online, Rio de Janeiro, v. 7, n. 1, p. 1967-1974, 2015.

PIMENTE, E. D. C.; LUZ, G. S.; SCHIAVON, G. B.; PELlOSO, S. M.; CARVALHO, M. D. B. Teste do pezinho: a humanização do cuidado e do profissional. Revista Mineira de Enfermagem, Belo Horizonte, v. 14, n. 1, p. 25-28, 2010.

SALLES, M.; SANTOS, I. M. M. O conhecimento das mães acerca do teste do pezinho em uma unidade básica de saúde. Revista de Pesquisa: Cuidado é Fundamental Online, Rio de Janeiro, v. 1, n. 1, p. 5964, 2009.

SANTOS, E. C.; GAÍVA, M. A. M.; SANTOS, J. G.; ABUD, S. M. O conhecimento de puérperas sobre a triagem neonatal. Cogitare Enfermagem, Curitiba, v. 16, n. 2, p. 282-288, 2011.

STREFLING, I. S. S.; MONFRIM, X. M.; LUNARDI FILHO, W. D.; CARVALHO, K. K.; AZEVEDO, A. L. S. Conhecimento sobre triagem neonatal e sua operacionalização. Cogitare Enfermargem, Curitiba, v. 19, n. 1, p. 27-33, 2014.

TASSINARY, R. F.; HAHN, G. V. Intervenções de enfermagem para o alívio da dor em recémnascidos. Pediatria Moderna, São Paulo, v. 49, n. 6, p. 219-226, 2013.

Recebido em:20 maio 2016 Aceito em: 7 nov. 2016 